\title{
Publisher's announcement
}

๑ Springer Science+Business Media, LLC, part of Springer Nature 2019

The publisher and the editor-in-chief of International Journal of Speech Technology would like to inform its readers that the September issue publishes contributions for three special issues. The titles of the special issues, names of the guest editors and articles concerned are listed below.

\section{Special issue on 'Emotion Recognition in Speech', guest editor Imen Trabelsi}

Emotions recognition: different sets of features and models

C. Jeyalakshmi http://doi.org/10.1007/s10772-018-9533-6

Improving the performance of the speaker emotion recognition based on low dimension prosody features vector

Ashishkumar Gudmalwar https://doi.org/10.1007/s1077 2-018-09576-4

Enhanced speech emotion detection using deep neural networks

S. Lalitha https://doi.org/10.1007/s10772-018-09572-8

Emotional speech analysis using harmonic plus noise model and Gaussian mixture model

Jang Singh https://doi.org/10.1007/s10772-018-9549-y

Prosodic Transformation in Vocal Emotion Conversion for multi-lingual scenarios—a pilot study

Deepa Gupta https://doi.org/10.1007/s10772-019-09626-5

Classifying females' stressed and neutral voices using acoustic-phonetic analysis of vowels: an exploratory investigation with emergency calls

Lauri Tavi https://doi.org/10.1007/s10772-018-09574-6

Automatic music emotion classification using hashtag graph

Deepti Chaudhary https://doi.org/10.1007/s10772-01909629-2

\section{Special issue on 'Speech and Next Generation Computing', guest editors Med Salim Bouhlel and Neelanjan Dey}

Convolutional support vector machines for speech recognition
Vishal Passricha https://doi.org/10.1007/s10772-018-09584 $-4$

Reference free speech quality estimation for diverse data condition

Nirupam Shome https://doi.org/10.1007/s10772-018-9537-2

A hybrid approach for Arabic lemmatization

Mohamed Boudchiche https://doi.org/10.1007/s1077 2-018-9528-3

Dual estimation based vocal tract shape computation

Subhasmita Sahoo https://doi.org/10.1007/s 1077 2-018-9538-1

Speech signal analysis as an alternative to spirometry in asthma diagnosis: investigating the linear and polynomial correlation coefficient

John Kutor https://doi.org/10.1007/s10772-019-09608-7

\section{Special issue on 'Artificial Intelligence and Automatic Speech Processing', guest editor Hanane Tebbi}

Unsupervised learning blocking keys technique for indexing Arabic entity resolution

Marwah Alian https://doi.org/10.1007/s1077 2-018-9489-6

Speech enhancement by combining spectral subtraction and minimum mean square error-spectrum power estimator based on zero crossing

Thimmaraja Yadava https://doi.org/10.1007/s1077 2-018-9506-9

A new hybrid approach for speech synthesis: application to the Arabic language

Hanane Tebbi https://doi.org/10.1007/s10772-018-9499-4

Amy Neustein Editor-in-Chief 